\title{
A vantagem em casa no futebol: comparação entre Copa Libertadores da América e UEFA Champions League
}

CDD. 20.ed. 796.073

796.33

http://dx.doi.org/10.1590/1807-55092014000200283

\section{Resumo}

0 objetivo desse estudo foi comparar a vantagem em casa (VC) entre Copa Libertadores da América (CLA) e UEFA Champions League (UCL). Foram analisadas todas as partidas da fase de grupos ( $n=1536)$ das temporadas 2004 a 2011. Para quantificar a VC, foi utilizada a metodologia de aproveitamento percentual de pontos proposta por PollARD ${ }^{3}$, sendo considerado VC valores maiores que $50 \%$. Houve diferença significativa $(p=0,010)$ para a VC na CLA (67,8 \pm 4\%) em relação a UCL (60,5 \pm 5\%). A magnitude das diferenças das médias (diferença média =0,73; IC 95\%: 0,020 a 0,127) foi grande $\left(\eta^{2}=0,35\right)$. Conclui-se que a VC foi maior na CLA do que na UCL nas temporadas analisadas e que fatores como altitude, distância de viagem, característica das torcidas e comportamento arbitral poderá ajudar a explicar esse fenômeno em futuros estudos.

PalAVRAS-Chave: Jogar em casa; Defesa territorial; Desempenho esportivo; Futebol.

\section{Introdução}

A Copa Libertadores da América (CLA) é a principal competição entre clubes de futebol das Américas. Sua primeira edição foi realizada em 1960 e organizada anualmente pela CONMEBOL (Confederação Sul-Americana de Futebol) envolvendo os campeóes e primeiros colocados de 11 países da América Latina ${ }^{1}$. Já a Liga dos Campeóes da UEFA (UCL) ou oficialmente UEFA Champions League, é organizada anualmente pela UEFA (União das Federaçôes Europeias de Futebol) desde 1955-56, e também é a mais importante competição de futebol na Europa envolvendo pelo menos um representante de cada uma das 53 federaçóes filiadas à UEFA ${ }^{2}$.

Além do nível técnico, diferentes característica de um desporto e de uma competição (i.e. sistema de disputa, premiação, condições geográficas das sedes/ locais) podem ser fatores a serem considerados pelas comissões técnicas no planejamento e assim determinantes no resultado final dos confrontos jogo a jogo. Um fato bem documentado para vários esportes coletivos e individuais é a vantagem de se jogar em casa (VC) em uma competição ${ }^{3-7}$. Diversos autores ${ }^{3,8-12}$ têm sugerido possibilidades que podem conferir essa vantagem às equipes mandantes como a torcida; familiaridade com o campo de jogo; favorecimento da arbitragem; deslocamento das equipes visitantes; tipo de bola fornecida pelo mandante, ou até mesmo níveis aumentados de testosterona nos jogadores das equipes mandantes como indicador de defesa territorial. Portanto, a VC pode caracterizar uma competição e interferir no balanço competitivo dela ${ }^{13}$, alterando comportamentos táticos em função de adversários que historicamente apresentem vantagem quando joga em casa, independente do nível técnico da equipe, ou mesmo modificando rotinas em função de viagens.

Vários estudos têm demonstrado o comportamento da VC em diferentes campeonatos de futebol como nas duas principais divisóes do futebol brasileiro $^{14}$ e nos principais campeonatos nacionais do mundo ${ }^{7,15-16}$. Na Inglaterra, por exemplo, o comportamento da VC no futebol é conhecido em diferentes épocas e divisóes ${ }^{6,17-18}$. Atualmente encontram-se valores variando de 60-65\%, com diferenças não significativas entre a primeira e segunda divisão nos países da Europa ${ }^{6,12,19-20}$. No Brasil, os estudos de Silva e Moreira ${ }^{16}$ e Pollard et al..$^{21}$ apontam que os valores encontrados $(-65 \%)$ são maiores que os das principais ligas nacionais da Europa. 
Apesar dessas documentaçôes, não há dados e comparaçōes disponíveis na literatura sobre VC nos dois principais campeonatos continentais no mundo (Copa Libertadores de América vs. UEFA Champions League). Suas formas de disputas são diferentes aos estudos supracitados que analisaram competiçóes nacionais com pontos corridos ${ }^{6,12,16,19,21}$, o que poderá ocasionar diferentes índices de VC na CLA e UCL em função de abordagens táticas e de estratégias de jogo particulares para competiçôes com esse formato de disputa.

\section{Métodos}

O delineamento proposto para o presente estudo foi pensado para que futuros estudos tenham informações sobre o fenômeno da VC e desta maneira orientar na formulação de hipóteses. Num primeiro momento foi adotado um caráter descritivo, com análise da VC por percentual de aproveitamentos de pontos. Num segundo momento a pesquisa assume uma característica inferencial, adotando teste de hipótese para testar a possibilidade de a VC ser maior na CLA em relação a UCL. Portanto, tem se como variável independente as competiçóes (CLA e UFC) e a como variável dependente a VC (\%).

Todos os modelos de disputa analisados contavam com o mesmo sistema de promoção para a próxima fase. Pelo formato das duas competiçôes, em sua fase de grupos, todos os times se enfrentavam duas vezes, em jogos de turno e returno, com mando de campo sendo revezado de modo que cada time jogasse uma vez em seu estádio e outra vez no estádio de outro adversário do grupo. Por questóes de intervalo temporal (aproximadamente dois meses) entre a fase de grupo e eliminatória na UCL, foi considerado para as análises apenas as fases de grupo, intencionando assim controlar vieses de capacidade competitiva das equipes (reforço na janela de transferência de dezembro) e de climas, que poderiam se alterar entre uma fase e outra na competição europeia.

\section{Amostra}

Foram analisadas 1536 partidas em casa da fase de grupos da Copa Libertadores da América e da UEFA Champions League (TABELA 1). Na CLA
Assim, o objetivo do presente estudo foi comparar a VC da CLA e UCL analisando o percentual de pontos obtidos em casa. Em virtude de apontamentos anteriores ${ }^{15}$ é esperado que a VC na CLA seja maior que na UCL em virtude de características geográficas próprias da América do Sul, que inclusive caracterizam marcantemente a VC de competiçóes em alguns países nesse continente em relação as competiçôes dos principais países europeus.

foram utilizadas as temporadas de 2004 a 2011 e na UCL de 2004/2005 à 2011/2012. Todas adotaram o critério de três pontos por vitória, um ponto para empate e zero ponto para derrota. As tabelas das ligas foram consultadas via internet, no site www. soccerway.com. Este site tem sido utilizado em estudos prévios para cálculo da $\mathrm{VC}^{14-16,21}$.

\section{Procedimentos}

Para quantificação da VC, foi utilizada a metodologia do aproveitamento percentual de pontos em casa, proposta por Pollard ${ }^{3}$. Considera-se que há VC quando o aproveitamento é maior que 50\%. Para ampliar as possibilidades de identificação de características regionais, uma análise descritiva dos comportamentos individuais dos clubes em relação a VC seguindo os mesmos critérios de Pollard ${ }^{3}$ foi realizada.

\section{Análise dos resultados}

Os dados são apresentados como média \pm desviopadrão. Pressuposições de normalidade foram verificadas por Shapiro-Wilks test. Para comparação entre CLA e UCL, empregou-se o Student's t-test. Effect sizes $\left(\eta^{2}\right)$ foram calculados para estimar a magnitude das diferenças e valores de 0,01, 0,06 e maiores que 0,14 foram considerados como pequeno, médio e grande, respectivamente ${ }^{22}$. Todas as análises estatísticas foram realizadas pelo "software" Statistical Package for the Social Sciences (SPSS ${ }^{\circ}$ 17 for Windows, Chicago, IL, EUA). O nível de significância adotado foi de 5\%. 
TABELA 1 - Jogos analisados na Copa Libertadores da América e na UEFA Champions League entre as temporadas de 2004 a 2011.

\begin{tabular}{lccccc}
\hline & \multicolumn{3}{c}{ Copa Libertadores da América } & \multicolumn{3}{c}{ UEFA Champions League } \\
Temporada & Jogos & Equipes & Temporada & Jogos & Equipes \\
\hline 2004 & 96 & 32 & $2004 / 2005$ & 96 & 32 \\
2005 & 96 & 32 & $2005 / 2006$ & 96 & 32 \\
2006 & 96 & 32 & $2006 / 2007$ & 96 & 32 \\
2007 & 96 & 32 & $2007 / 2008$ & 96 & 32 \\
2008 & 96 & 32 & $2008 / 2009$ & 96 & 32 \\
2009 & 96 & 32 & $2009 / 2010$ & 96 & 32 \\
2010 & 96 & 32 & $2010 / 2011$ & 96 & 32 \\
2011 & 96 & 32 & $2011 / 2012$ & 96 & 32 \\
Total & 768 & & Total & 768 & \\
\hline
\end{tabular}

\section{Resultados}

Os resultados mostraram que o efeito $\mathrm{VC}$ existe nas duas competiçóes, sendo superiores a 50\% (CLA $=67,8 \pm 4 \%$ e UCL $=60,5 \pm 5 \%)$. Um teste $\mathrm{t}$ para medidas independentes foi realizado para comparar a VC na Copa Libertadores de América e na UEFA Champions League. Houve diferença significativa $(\mathrm{p}=0,010)$ na VC para a CLA em relação a UCL (FIGURA 1). A magnitude das diferenças das médias (diferença média = 0,73; IC 95\%: 0,020 a $0,127)$ foi grande $\left(\eta^{2}=0,35\right)$.
Análises descritivas da VC dos clubes (TABELA 2) revelaram que os de regióes com altitude (i.e. clubes do México) e/ou de regióes que podem provocar maiores distâncias de deslocamentos (i.e. clubes do México e Brasil) obtiveram maiores índices de VC na CLA. Para a UEFA nota-se, clube a clube, que o comportamento da VC tende a ser com menor índice, e quando são notados índices maiores percebe-se que eles pertencem a clubes mais tradicionais.

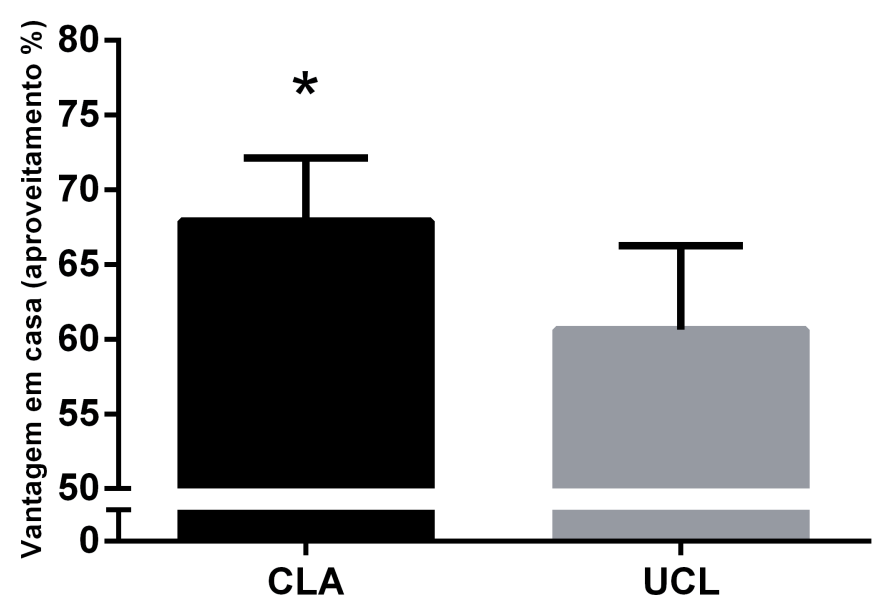

Valores da VC são expressos em média \pm desvio-padrão.

* Significativamente diferente $(p<0,05)$.

FIGURA 1 - Vantagem em casa (VC) registrada para a Copa Libertadores da América e UEFA Champions League entre as temporadas de 2004 a 2011. 
TABELA 2 - Comportamento individual da vantagem em casa por clubes na Copa Libertadores da América e na UEFA Champions League entre as temporadas de 2004 a $2011(n=8)$.

\begin{tabular}{|c|c|c|c|c|c|c|}
\hline \multirow{3}{*}{$\begin{array}{l}\mathrm{N}=\text { número de tempora- } \\
\text { das participantes; } \\
\text { VC = Vantagem de jogar } \\
\text { em casa. }\end{array}$} & \multicolumn{3}{|c|}{ Copa Libertadores da América } & \multicolumn{3}{|c|}{ UEFA Champions League } \\
\hline & Equipes & $\mathbf{N}$ & VC & Equipes & $\mathbf{N}$ & VC \\
\hline & Bayer Leverkusen/ALE & 2 & 100 & Atlas/MEX & 1 & 100 \\
\hline & Tottenham/ING & 1 & 100 & Boca Júniors/ARG & 4 & 100 \\
\hline & Fiorentina/ITA & 1 & 100 & Jaguares/MEX & 1 & 100 \\
\hline & Monaco/ITA & 1 & 100 & Necaxa/MEX & 1 & 100 \\
\hline & Sevilla/ESP & 2 & 94 & Pachuca/MEX & 1 & 100 \\
\hline & Arsenal/ING & 8 & 92 & Cruzeiro/BRA & 5 & 98 \\
\hline & Chelsea/ING & 8 & 92 & Internacional/BRA & 4 & 97 \\
\hline & Napoli/ITA & 1 & 88 & Flamengo/BRA & 3 & 96 \\
\hline & København/DIN & 2 & 88 & Santos/BRA & 5 & 95 \\
\hline & AEK/GRE & 1 & 88 & Corinthians/BRA & 2 & 94 \\
\hline & Manchester City/ING & 1 & 88 & Grêmio/BRA & 3 & 92 \\
\hline & Real Madrid/ESP & 8 & 87 & São Paulo/BRA & 7 & 91 \\
\hline & Barcelona/ESP & 8 & 85 & Estudiantes/ARG & 5 & 91 \\
\hline & Juventus/ITA & 4 & 84 & Goiás/BRA & 1 & 88 \\
\hline & Bayern München/ALE & 7 & 82 & Santa Fe/COL & 1 & 88 \\
\hline & Celtic/ESC & 4 & 80 & Newell's old boys/ARG & 1 & 88 \\
\hline & Schalke 04/ALE & 3 & 79 & Deportivo Quito/EQU & 2 & 88 \\
\hline & Manchester United/ING & 8 & 78 & Santo André/BRA & 1 & 88 \\
\hline & Internazionale/ITA & 8 & 76 & Santos Laguna/MEX & 1 & 88 \\
\hline & Olympique Lyon/FRA & 8 & 75 & Coritiba/BRA & 1 & 88 \\
\hline & Liverpool/ING & 6 & 74 & América/MEX & 5 & 87 \\
\hline & Milan/ITA & 7 & 72 & Vélez Sarsfield/ARG & 5 & 86 \\
\hline & Unirea Urziceni/ROM & 1 & 71 & Fluminense/BRA & 2 & 86 \\
\hline & Valencia/ESP & 8 & 71 & River Plate/ARG & 6 & 85 \\
\hline & Anorthosis/GRE & 1 & 71 & LDU Quito/EQU & 7 & 83 \\
\hline & Olympiakos Piraeus/GRE & 4 & 71 & Universidad do Chile/CHI & 3 & 81 \\
\hline & Porto/POR & 7 & 69 & Universitário/PER & 2 & 79 \\
\hline & Werder Bremen/ALE & 5 & 68 & Guadalajara/MEX & 4 & 78 \\
\hline & Beşiktaş/TUR & 2 & 67 & Junior/COL & 2 & 77 \\
\hline & $\mathrm{PSV} / \mathrm{HOL}$ & 5 & 64 & Peńarol/URU & 2 & 77 \\
\hline & Benfica/POR & 5 & 64 & Audax Italiano/CHI & 2 & 77 \\
\hline & Roma/ITA & 5 & 64 & Medelin/COL & 3 & 76 \\
\hline & Bordeaux/FRA & 3 & 61 & Nacional/URU & 8 & 75 \\
\hline & Rubin Kazan'/RUS & 2 & 60 & Once Caldas/COL & 4 & 72 \\
\hline & Atlético Madrid/ESP & 2 & 58 & Sporting Cristal/PER & 3 & 72 \\
\hline & Zenit/RUS & 4 & 58 & Cúcuta Deportivo/COL & 2 & 71 \\
\hline & Fenerbahçe/TUR & 4 & 57 & Morelia/MEX & 1 & 71 \\
\hline & Lille/FRA & 3 & 57 & Monterrey/MEX & 1 & 71 \\
\hline & Shakhtar Donetsk/UCR & 6 & 55 & Everton/CHI & 1 & 71 \\
\hline & Sport Lisboa/POR & 4 & 54 & Cerro/PAR & 1 & 71 \\
\hline & Ajax/HOL & 4 & 54 & Paulista/BRA & 1 & 71 \\
\hline
\end{tabular}


TABELA 2 - Comportamento individual da vantagem em casa por clubes na Copa Libertadores da América e na UEFA Champions League entre as temporadas de 2004 a $2011(n=8)$ (continuação).

\begin{tabular}{|c|c|c|c|c|c|}
\hline \multicolumn{3}{|c|}{ Copa Libertadores da América } & \multicolumn{3}{|c|}{ UEFA Champions League } \\
\hline Equipes & $\mathbf{N}$ & VC & Equipes & $\mathbf{N}$ & VC \\
\hline Villarreal/ESP & 3 & 53 & Quilmes/ARG & 1 & 71 \\
\hline Wolfsburg/ALE & 1 & 50 & Real Potosí/BOL & 2 & 69 \\
\hline Udinese/ITA & 1 & 50 & Independiente/ARG & 2 & 69 \\
\hline Panathinaikos/GRE & 4 & 50 & Juan Aurich/PER & 1 & 67 \\
\hline Hapoel Tel Aviv/ISR & 1 & 50 & Paraná/BRA & 1 & 67 \\
\hline $\mathrm{AZ} / \mathrm{HOL}$ & 1 & 50 & Defensor Sporting/PAR & 2 & 67 \\
\hline Trabzonspor/TUR & 1 & 50 & Boyacá Chicó/COL & 1 & 67 \\
\hline Twente/HOL & 1 & 50 & Sport Recife/BRA & 1 & 67 \\
\hline Club Brugge/BEL & 1 & 50 & Oriente PetroleroBOL & 1 & 67 \\
\hline Standard Liege/FRA & 1 & 50 & Racing Clube/ARG & 1 & 67 \\
\hline Lazio/ITA & 1 & 50 & Gimnasia/ARG & 1 & 67 \\
\hline Galatasaray/TUR & 1 & 50 & Banfield/ARG & 3 & 67 \\
\hline Genk/HOL & 1 & 50 & Toluca/MEX & 1 & 67 \\
\hline Slavia Praha/REP ZCH & 1 & 50 & Deportivo Cuenca/PER & 4 & 67 \\
\hline Olympiakos/GRE & 2 & 50 & Arsenal/ARG & 1 & 67 \\
\hline Borussia Dortmund/ALE & 1 & 50 & Atlético PR/BRA & 1 & 67 \\
\hline $\mathrm{AaB} / \mathrm{DIN}$ & 1 & 50 & Barcelona/EQU & 1 & 67 \\
\hline Olympique Marseille/FRA & 7 & 47 & Libertad/PAR & 8 & 66 \\
\hline CSKA Moskva/RUS & 5 & 43 & Tigres UANL/MEX & 2 & 66 \\
\hline Rangers/ESC & 4 & 43 & Colo Colo/CHI & 6 & 65 \\
\hline Stuttgart/ALE & 2 & 41 & Deportes Tolima/COL & 3 & 63 \\
\hline APOEL/TUR & 2 & 40 & Bolivar/BOL & 5 & 63 \\
\hline Dynamo Kyiv/UCR & 5 & 36 & The Strogest/BOL & 3 & 62 \\
\hline Hamburger SV/ALE & 1 & 33 & San Lorenzo/ARG & 3 & 61 \\
\hline Thun/SUI & 1 & 33 & Atlético Nacional/COL & 2 & 60 \\
\hline Real Betis/ESP & 1 & 33 & Cienciano/PER & 4 & 58 \\
\hline Basel/SUI & 3 & 33 & Palmeiras/BRA & 3 & 57 \\
\hline Auxerre/FRA & 1 & 33 & Caracas/VEN & 8 & 54 \\
\hline PSG/FRA & 1 & 33 & Alianza Lima/PER & 4 & 54 \\
\hline CFR Cluj/ROM & 2 & 32 & El Nacional/EQU & 3 & 51 \\
\hline Spartak Moscow/RUS & 2 & 31 & Universidad San Martin/PER & 4 & 50 \\
\hline Viktoria Plzen/REP & 1 & 29 & Universidad Católica/CHI & 4 & 50 \\
\hline Anderlecht/BEL & 3 & 29 & Danúbio/URU & 2 & 50 \\
\hline Petržalka/ESL & 1 & 29 & Lanús/ARG & 3 & 50 \\
\hline Rosenborg/NOR & 3 & 25 & León de Huánuco/PER & 1 & 50 \\
\hline Maccabi Haifa/ISR & 2 & 25 & San Luis/MEX & 2 & 50 \\
\hline Sparta Praha/REP & 2 & 21 & Deportivo Táchira/VEN & 4 & 50 \\
\hline Deportivo La Coruña/ESP & 1 & 13 & Sportivo Luqueño/PAR & 1 & 50 \\
\hline Zürich/SUI & 1 & 13 & Rocha FC/URU & 1 & 50 \\
\hline Bursaspor/TUR & 1 & 13 & Union Española/CHI & 2 & 50 \\
\hline BATE/RUS & 2 & 13 & Godoy Cruz/ARG & 1 & 50 \\
\hline
\end{tabular}

continua

Rev Bras Educ Fís Esporte, (São Paulo) 2014 Abr-Jun; 28(2):283-92 • 287 
TABELA 2 - Comportamento individual da vantagem em casa por clubes na Copa Libertadores da América e na UEFA Champions League entre as temporadas de 2004 a $2011(n=8)$ (continuação).

\begin{tabular}{|c|c|c|c|c|c|}
\hline \multicolumn{3}{|c|}{ Copa Libertadores da América } & \multicolumn{3}{|c|}{ UEFA Champions League } \\
\hline Equipes & $\mathbf{N}$ & VC & Equipes & $\mathbf{N}$ & VC \\
\hline Žilina/ALE & 1 & 0 & Rosário Central/ARG & 2 & 50 \\
\hline Partizan/SER & 1 & 0 & São Caetano/BRA & 1 & 50 \\
\hline Levski Sofia/BUL & 1 & 0 & Fénix/URU & 1 & 50 \\
\hline Dinamo Zagreb/CRO & 1 & 0 & Olímpia/PAR & 1 & 50 \\
\hline Otelul Galati/ROM & 1 & 0 & Cerro Porteño/PAR & 5 & 49 \\
\hline Debrecen/HUN & 1 & 0 & Emelec/QUE & 3 & 45 \\
\hline Steaua Bucareste/ROM & 3 & 0 & Nacional Assunción/PAR & 2 & 41 \\
\hline \multirow[t]{17}{*}{ Rapid Wien/AUS } & 1 & 0 & Jorge Wilstermann/BOL & 2 & 40 \\
\hline & & & Deportivo Cali/COL & 2 & 40 \\
\hline & & & UA Maracaibo/VEN & 4 & 35 \\
\hline & & & Cobreloa/CHI & 2 & 35 \\
\hline & & & San José/BOL & 1 & 33 \\
\hline & & & Argentinos Juniors/ARG & 1 & 33 \\
\hline & & & Olmedo/QUE & 1 & 33 \\
\hline & & & Universitário de Sucre/BOL & 1 & 29 \\
\hline & & & América de Cali/COL & 1 & 29 \\
\hline & & & Bolognesi/PER & 1 & 29 \\
\hline & & & Guaraní/PAR & 3 & 21 \\
\hline & & & Deportivo Petare/VEN & 1 & 13 \\
\hline & & & Deportivo Pasto/COL & 1 & 13 \\
\hline & & & Pumas UNAM/MEX & 1 & 13 \\
\hline & & & Universidad de Concepción/PAR & 1 & 13 \\
\hline & & & Aurora/BOL & 1 & 0 \\
\hline & & & Blooming/BOL & 1 & 0 \\
\hline
\end{tabular}

\section{Discussão}

O presente estudo investigou a VC na CLA e UCL através do percentual de aproveitamentos de pontos em casa. Resultados mostraram que um efeito de VC existe nas duas competiçóes, sendo, no entanto maior na CLA quando comparada à UCL nas temporadas de 2004/2005 a 2011/2012, corroborando hipótese especulada para o presente estudo.

Vários fatores podem ser levados em consideração para explicar esta maior VC na CLA. Incialmente pode se considerar que condição geográfica diferenciada pôde conferir maior VC na América do Sul para sua principal competição (CLA) no período estudado (2004 a 2011). Como sugerido por POLLARD ${ }^{15}$, principalmente na regiâo andina, há dificuldades dos visitantes por enfrentamentos de grandes altitudes, podendo interferir na VC da CLA.
Nesses locais as equipes visitantes são susceptíveis a terem dificuldade para adaptar-se à altitude (hipóxia hipobárica), afetando adversamente seu desempenho (físico e técnico). Já por outro lado quando as equipes de grandes altitudes visitam outras ao nível do mar, essas podem não sofrer queda do desempenho físico.

Os dados do presente trabalho (TABELA 2) sugerem que equipes que possuem seus estádios em grande altitude apresentam grandes VC, com é o caso de LDU de Quito e Deportivo Quito, que possuem VC de $83 \%$ e $88 \%$ respectivamente. Esses clubes utilizam os estádios "Estádio da Liga Deportiva Universitária" (2.774 metros de altitude) e "Estádio Olímpico de Atahualpa" (2850 metros de altitude) respectivamente. Vale ressaltar que isso 
assume um peso de efeito competitivo importante para a primeira fase da competição como estudada no presente estudo. Porém, esse efeito ainda é insignificante quando se avalia o nível técnico e número de títulos dessas equipes ao longo da história da CLA frente a clubes brasileiros e argentinos. Afinal equipes com maior poderio competitivo tendem a vencer independente do local do jogo.

Distância viajada poderia ser outra plausível explicação onde equipes mexicanas e brasileiras estão com altos índices de VC (> 90\%; TABELA 2) e são praticamente as que mais provocam deslocamentos em seus adversários. Diferentemente na Europa, alguns estudos têm demonstrado que a influência do deslocamento não explicou as taxas de $\mathrm{VC} \mathrm{em}$ competiçôes domésticas ${ }^{23-24}$. No entanto, quando são comparados dois campeonatos continentais como no caso desse estudo, em ambos haverá grandes deslocamentos para as equipes visitantes, porém os países do continente europeu possuem uma infraestrutura de transporte muito mais desenvolvida do que a dos países sul-americanos, o que torna as viagens dos clubes na UCL menos demoradas, mais seguras e confortáveis. Portanto, a nível continental isso pode diminuir o desgaste físico provocado pelas viagens e a queda do rendimento ao final das partidas como apontado por alguns estudos ${ }^{21,25}$. Essas viagens que provocam menos cansaço, ainda podem colaborar para uma melhor percepção e estado psicológico para enfrentamento em jogos fora de casa ${ }^{26-27}$.

Um exemplo que pode reforçar essa hipótese do maior deslocamento de visitantes e o impacto positivo na VC para os times sul americanos em comparação com os europeus é um estudo desenvolvido sobre o campeonato Brasileiro da primeira divisão ${ }^{21}$. Esses pesquisadores observaram que a distância de deslocamento entre as equipes provocavam um efeito pequeno, mas significativo sobre o resultado de um jogo $(\mathrm{p}<0,01)$ resultando em 0,115 de um gol a favor da equipe da casa para cada 1.000 quilômetros percorridos pela equipe visitante. Em recente estudo, LAGo-PeÑAS et al. ${ }^{28}$ observaram que a distância de deslocamento dos jogadores em campo foi explicada pela localizaçáo do jogo. Os jogadores das equipes que jogam em casa possuem um maior deslocamento total em campo durante partidas do campeonato espanhol nas temporadas de 2005-2006, demonstrando que o fator casa pode criar predisposiçáo física para o jogo.

Outro fator evidenciado no presente estudo que pode corroborar a hipótese de efeitos de viagens dos visitantes na VC pode ser observado pelo desempenho das equipes mexicanas na CLA, já que o México é o país que fica mais distante dentre os países que disputam a CLA. Elas apresentam grande VC no período estudado, como América do México (100\%), Pachuca (100\%), Tigres de La UANL (86\%) e Chivas de Guadalajara (75\%) (TABELA 2). Assim essa grande VC dessas equipes pode ser em parte explicada pelas longas viagens que as equipes visitantes devem fazer. O América do México, com $100 \%$ de aproveitamento em casa possui dois tipos de vantagem a seu favor, pois além da longa distância ainda joga na altitude. O seu estádio "Estádio Azteca" fica a 2.240 metros de altitude.

Além do aspecto geográfico, as características da torcida nos dois continentes são bem diferentes. A média de presença de torcedores nos principais campeonatos nacionais na Europa é maior do que nas ligas nacionais sul-americanas. Enquanto nos campeonatos europeus são vendidos em média 20.000 ingressos por jogo, no Brasil são 12.000, na Argentina são 10.600 e no Chile $4.800^{29}$, o que na teoria elevaria a VC na UCL. Porém outro fator relacionado à torcida que parece aumentar a VC na CLA independente dos números presentes nos estádios sul-americanos é a densidade, o fanatismo, barulho e hostilidade, inclusive contra árbitros e assistentes, sendo assim um indicador mais forte que somente o tamanho dela ${ }^{6}$. Um exemplo para isso é a equipe do Boca Juniors da Argentina que reconhecidamente faz de seu estádio "La Bombonera" um verdadeiro "caldeirão", possuindo uma VC de $100 \%$ nas fases classificatórias no período estudado, demonstrando assim que a torcida pode ser um dos fatores influenciadores da VC. Isso pode ser estendido para diversos clubes argentinos que possuem altos índices de VC (> 85\%; TABELA 2) demonstrando que características de torcidas e comportamento típico de jogadores frente à equipe de arbitragem podem provocar efeito positivo de VC para eles.

A torcida local pode exercer forte influência na pré-disposição para a realizaçáo de um bom jogo segundo jogadores profissionais da liga ingles ${ }^{26}$, além de prejudicar psicologicamente a equipe adversária e exercer também uma forte influência sobre as decisóes do árbitro através da "pressão" e intimidação imposta à comissão de arbitragem. Tem sido relatado o aumento do número de faltas ${ }^{4}$ e pênaltis ${ }^{11,18}$ marcados a favor da equipe da casa, e um maior número de cartóes amarelos aplicados aos jogadores das equipes visitantes $^{11,30}$, o que pode indicar uma inclinação das decisōes dos árbitros a favor do mandante.

Não há dados sobre a influência arbitral na CLA, no entanto pela estrutura de estádios, comportamentos de torcida e conduta até mais permissiva por parte de organizadores, espera-se que o clima hostil na 
CLA potencialize o efeito do jogar em casa nessa competição. Levantamentos dessas informações são necessários para corroborar e rejeitar essa hipótese de que a arbitragem é tendenciosa e factível a influência do contexto do jogo como é especulado.

Locais geográficos remotos podem estimular uma defesa territorial mais ofensiva e hostil ${ }^{7}$, de forma instintiva e orgânica, sendo observado, por exemplo, maiores taxas de testosterona nos mandantes ${ }^{8}$. Nesse sentido a maior presença de jogadores locais nos clubes da CLA poderia aumentar a percepção de defesa territorial. Isso pode ser um fator diferencial dos sul americanos, pois quando se observa a composição de elencos dos principais clubes europeus, percebe-se a predominante presença de jogadores de todas as partes do mundo, o que poderia diminuir a percepção de defesa territorial na UCL, ocasionando uma menor taxa de VC em relação à CLA.

Outro fator fundamental para explicar a maior VC no futebol sul-americano pode ser a familiaridade com o campo de jogo, considerada pelos técnicos e atletas como aspecto mais importante na $\mathrm{VC}^{31}$. É notório observar que há diferenças nas dimensôes da área do campo de jogo e nas condiçóes do gramado entre os estádios na CLA e isso podem estar trazendo implicaçôes táticas e de demandas de carga fisiológica diferentes, acarretando prejuízo no desempenho dos visitantes na competição interclubes sul americana. $\mathrm{Na}$ Europa os regulamentos das competições são mais severos para adequar as condiçóes pré-estabelecidas, levando assim a uma maior padronizaçáo. Da mesma forma fatores manipulativos no local do jogo na CLA também podem ocorrer e dificultar ainda mais os adversários. A interferência no estado do gramado minutos antes da partida, ausência de condiçóes e horários adequados para o reconhecimento do campo de jogo e aquecimento pré-jogo são exemplos disso. Isso foi alertado por SiLva e MoREIRA $^{16}$ para a maior VC no campeonato brasileiro frente às principais ligas europeias.

Cabe aqui destacar, que a metodologia aplicada para calcular a VC através do percentual de aproveitamento de pontos tem suas desvantagens, uma vez que essa metodologia não leva em consideração a qualidade das equipes analisadas, sendo que provavelmente, quando são pareadas duas equipes de qualidade muito diferentes, a melhor vença o jogo mesmo este sendo em casa ou não ${ }^{16}$.

Dessa forma, destaca-se a importância de que novas pesquisas analisem a VC na CLA e UCL levando em consideraçáo as qualidades das equipes e as particularidades do seu campo e local de jogo. Estabelecimentos de efeitos causais e percepção por parte de jogadores são pontos que poderão elucidar melhor o entendimento e atribuiçóes da VC no futebol sul americano e europeu em futuros estudos.

Conclui-se que há uma maior VC na Copa Libertadores da América quando comparada à UEFA Champions League. Portanto na CLA, durante a fase de grupos, a vitória em casa é mais importante para a classificação das equipes para a próxima fase da competição. Poucas pesquisas foram desenvolvidas no sentido de análise da VC nessas competiçōes, mas características geográficas, torcida e comportamento arbitral parecem indicar caminhos para o entendimento da maior VC na competição sul americana.

No sentido prático, cabe a COMENBOL coibir comportamentos hostis que prejudicam equipes visitantes e que poderiam também influenciar a decisão arbitral ampliando a VC. Maior padronizaçáo no campo de jogo no que tange as condiçóes e dimensóes dos gramados poderão ser medidas adotadas por essa confederaçáo continental visando maior equilíbrio competitivo entre os participantes da CLA.

Assim a VC pode refletir certo grau de certeza acerca do resultado da partida ou da competição, com importante impacto no futebol visto como negócio. Deste modo, controlando fatores externos ao jogo como os citados acima, há maior possibilidade de aumentar o equilíbrio competitivo na CLA, legitimar resultados e assim ampliar a captação de recursos financeiros por parte dos clubes e gerar maior interesse do público.

\section{Notas}

O autor Lucas Rios Drummond é aluno do Programa de Pós-Graduação em Ciências Biológicas: Fisiologia e Farmacologia, do Instituto de Ciências Biológicas, da Universidade Federal de Minas Gerais.

O autor Filipe Rios Drummond é aluno do Programa de Pós-Graduação em Saúde e Nutrição, da Escola de Nutrição, da Universidade Federal de Ouro Preto.

O autor Cristino Diniz da Silva é aluno do Programa de Pós-Graduação em Ciências do Esporte, da Escola de Educação Física, Fisioterapia e Terapia Ocupacional, da Universidade Federal de Minas Gerais 


\section{Abstract}

The home advantage in soccer: comparision between Libertadores of American Cup and UEFA Champions League

The objective of this study was to compare the home advantage (HA) in Libertadores of American Cup (LAC) and UEFA Champions League (UCL). We analyzed all matches of group phase $(n=1536)$ from 2004 to 2011 seasons. To quantify the HA, we use the methodology of recovery percentage points, proposed by PollARD ${ }^{3}$, considered HA, values greater than $50 \%$. There were a significant difference $(p=0.010)$ between LAC $(67.8 \pm 4 \%)$ and UCL $(60.5 \pm 5 \%)$. The magnitude of the mean (mean difference $=0.73$, $95 \% \mathrm{Cl}: 0.020$ to 0.127$)$ was large $\left(\eta^{2}=0.35\right)$. We concluded that the HA was higher in LAC than in $\mathrm{UCL}$ in this seasons and the factors such as altitude, distance traveled fans characteristics and referee behavior it will be able to help to explain this phenomenon in future studies.

KEY WORDS: Home advantage; Territorial protection; Sports performance; Soccer.

\section{Referências}

1. CONMEBOL. Confederación Sudamericana de Fútbol. CONMEBOL 2012. Copa Libertadores. [citado 11 ago. 2012]. Disponível em: http://www.conmebol.com/.

2. UEFA. Union of European Football Associations. UEFA 2012. UEFA Champions League/História. [citado 11 ago. 2012]. Disponível em: http://pt.uefa.com/.

3. Pollard R. Home advantage in soccer: a retrospective analysis. J Sports Sci. 1986;4:237-48.

4. Nevill AM, Balmer NJ, Williams AM. The influence of crowd noise and experience upon refereeing decisions in football. Psychol Sport Exerc. 2002;3:261-72.

5. Clarke SR. Home advantage in the australian football league. J Sports Sci. 2005;23:375-85.

6?. Pollard R, Pollard G. Home advantage in soccer: a review of its existence and causes. Int J Soccer Sci. 2005;3:28-38.

7. Pollard R, Gómez MA. Home advantage in football in south-west europe: long term trends, regional variation and team differences. Eur J Sport Sci. 2009;9:341-52.

8. Neave N, Wolfson S. Testosterone, territoriality, and the 'home advantage'. Physiol Behav. 2003;78:269-75.

9. Tucker W, Mellalieu SD, James N, Taylor JB. Game location effects in professional soccer: a case study. Int JPerform Anal Sport. 2005;5:23-35.

10. Balmer NJ, Nevill AM, Lane AM, Ward P, Williams AM, Fairclough SH. Influence of crowd noise on soccer refereeing consistency in soccer. J Sports Behav. 2007;30:130-45.

11. Boyko RH, Boyko AR, Boyko MG. Referee bias contributes to home advantage in english premiership football. J Sports Sci. 2007;25:1185-94.

12. Dosseville FEM. Influence of ball type on home advantage in french professional soccer. Percept Mot Skills. 2007;104:347-51.

13. Forrest D, Beaumont J, Goddard J, Simmons R. Home advantage and the debate about competitive balance in professional sports leagues. J Sports Sci. 2005;23:439-45.

14. Almeida LG, Oliveira ML, Silva CD. Uma análise da vantagem de jogar em casa nas duas principais divisões do futebol profissional brasileiro. Rev Bras Educ Fís Esporte. 2011;25:49-54.

15. Pollard R. Worldwide regional variations in home advantage in association football. J Sports Sci. 2006;24:231-40.

16. Silva CD, Moreira DG. A vantagem em casa no futebol: comparaçáo entre o campeonato brasileiro e as principais ligas nacionais do mundo. Rev Bras Cineantropom Desempenho Hum. 2008;10:184-8.

17. Clarke S, Norman JM. Home ground advantage of individual clubs in english soccer. Statistician. 1995;44:509-21.

18. Nevill AM, Newell SM, Gale S. Factors associated with home advantage in english and scottish soccer matches. J Sports Sci. 1996;14:181-6.

19?. Pollard R, Pollard G. Long-term trends in home advantage in professional team sports in north america and england (1876-2003). J Sports Sci. 2005;23:337-50.

20. Gómez MA, Pollard R, Luis-Pascual J. Comparison of the home advantage in nine different professional team sports in Spain. Percept Mot Skills. 2011;113:150-6. 
Drummond LR, et al.

21. Pollard R, Silva CD, Medeiros NC. Home advantage in football in brazil: differences between teams and the effects of distance traveled. Rev Bras Futebol: Braz J Soccer Sci. 2008;1:3-10.

22. Cohen J. Statistical power analysis for the behavioral sciences. 2nd ed. Hillsdale: Lawrence Erlbaum; 1988

23. Courneya KS, Carron AV. Effects of travel and length of home stand/road trip on the home advantage. J Sport Exerc Psychol. 1991;13:42-9.

24. Smith DR, Ciacciarelli A, Serzan J, Lambert D. Travel and the home advantage in professional sports. Sociol Sport J. 2000;17:364-85.

25. Pollard R. Home advantage in football: a current review of an unsolved puzzle. Open Sports Sci J. 2008;1:12-4.

26. Waters A, Lovell G. An examination of the homefield advantage in a professional english soccer team from a psychological standpoint. Football Stud. 2002;5:46-59.

27. Renáta V, Dezso N. Psychological aspects of home advantage. Psychiatr Hung. 2006;21:422-9.

28. Lago-Peñas C, Rey E, Lago-Ballesteros J, Casáis L, Domínguez E. The influence of a congested calendar on physical performance in elite soccer. J Strength Cond Res. 2011;25:2111-7.

29. Deloitte Sport Business. Latin american football money league. 2006. [cited 2011 jul. 14]. Available from: http:// www.deloitte.com/assets/Dcom-Brazil/Local\%20Assets/Documents/Latin\%20American\%20Football\%20Money\%20 League.pdf.

30. Poulter DR. Home advantage and player nationality in international club football. J Sports Sci. 2009;27:797-805.

31. Wolfson S, Wakelin D, Lewis M. Football supporters' perceptions of their role in the home advantage. J Sports Sci. 2005;23:365-74.

Cristiano Diniz da Silva

ENDEREÇO

Faculdade de Viçosa

R. Gomes Barbosa, 870

Recebido para publicação: 08/01/2013

Viçosa - MG - BRASIL

Revisado: 19/04/2013

36570-OOo - Viçosa - MG - BRASIL
-mail: cristianodiniz.silva@gmail.com

Aceito: 03/02/2014

292 • Rev Bras Educ Fís Esporte, (São Paulo) 2014 Abr-Jun; 28(2):283-92 